\title{
Un operador de negación estricta para sistemas multivaluados
}

\section{Isis Urgell Menéndez}

Universidad de Buenos Aires, Argentina

Recibido el 20/1/18; aceptado el 5/3/2018.

\section{Resumen}

En el siguiente trabajo abordaré algunas cuestiones sobre el significado de la constante lógica "negación", y defenderé que posee dos rasgos fundamentales: el primero consiste en tener cierta prioridad formal y conceptual con respecto al resto de las constantes usuales conjunción y disyunción y el segundo en que su característica fundamental es la de ser un mecanismo de inversión hacia el opuesto entre valores de verdad. También discutiré algunos requisitos deseables para definir un operador de negación estricta o fuerte. Luego mostraré que es posible modelar un sistema cuatrivaluado a partir del conjunto FOUR añadiendo un operador que se comporte como una negación estricta y no clásica.

\section{A strict negation operator for multivalued systems}

\begin{abstract}
In this paper I will exam some issues about the meaning of the logical constant "negation". I will defend that it has two main features, one is being formally and conceptually prior to the other two basic logical connectives: conjunction and disjunction, and the other is having as a main character its ability to function as a switch of truth values, flip flopping one into the opposite. I will also argue for two other constraints one might ask for this type of operator to be called strict or strong. Finally, I show that is possible to design a multivalued logical system taking FOUR as a basis, and adding a strong yet non-classical negation to it.
\end{abstract}

\section{Palabras clave}

conectivos lógicos lógicas multivaluadas negación paradojas

\section{Keywords}

logical constants logical paradoxes multivalued logical systems negation 


\section{If we are by definition the animals that talk, we are ipso facto the animals that deny}

L. Horn

La negación es uno de los fenómenos más estudiados dentro del ámbito lingüístico y filosófico. Desde Aristóteles hasta nuestra época mucha tinta se ha derramado tratando de explicitar su funcionamiento y su lugar como mecanismo discursivo, fenómeno metafísico y operador lógico-matemático. En este trabajo realizaré un abordaje de la negación como conectivo lógico, trayendo a colación algunas tesis ya consideradas la ortodoxia sobre su significado y rol en un sistema formal, partiré del análisis de la negación clásica y pasaré a contrastarla con las negaciones no clásicas de sistemas multivaluados como $\mathrm{K}_{3}$, LP y FDE.

Mi objetivo es mostrar que, dada su capacidad de capturar diversos fenómenos fundamentales de nuestra práctica lingüística, epistémica e inferencial; y debido al rol que juega al momento de dilucidar la naturaleza de la relación de consecuencia lógica, así como la del resto de los conectivos de un sistema, la negación como operador lógico tiene una preeminencia por sobre los otros dos conectivos básicos del lenguaje lógico, la conjunción y la disyunción. Por otro lado, argüiré que, de todas sus características, debe destacarse como fundamental aquella que le permite realizar la función de inversión o "flip-flop" del valor de verdad otorgado, reflejando de manera formal el mecanismo de oposición entre elementos e inversión de contenidos con que suele asociarse preteóricamente.

\section{La negación como conectiva lógica fundamental}

La negación se ubica como una de las características fundamentales de la práctica lingüística y, por extensión, racional, de la especie humana. Según algunos autores (Cfr. Sebeok, 1962, citado en Horn, 1989).la posibilidad de negar podría ser la diferencia clave que explica la divergencia entre nuestro lenguaje y la comunicación animal. Desde que Parménides partió las aguas de la metafísica entre el ser y el no-ser, y Aristóteles definió el Principio de No Contradicción como principio rector de la racionalidad, la negación se ha configurado en la historia del pensamiento como un fenómeno particular que permea casi todas las áreas de nuestra práctica intelectual.

De manera general se suele caracterizar a la negación como un mecanismo de oposición semántica (Cfr. Horn, 2017). Esto es aproximadamente y de forma muy amplia, un mecanismo que permite enfrentar dos elementos opuestos sea en significado, contenido informacional o valor de verdad. De esta forma la negación puede entenderse desde su rol lingüístico en términos sintácticos -semánticos; en términos pragmáticos hablamos de "negación" (denial) como acto de habla; tiene un rol epistémico

1. Al respecto de la relación entre la definición de un operador de negación que modele correctamente la noción de rechazo y sus implicaciones metafísicas ver Rubin, $M \&$ Roffé, $A$.

2. Seguimos a Varzi, A. C.; Warglien, M. (2003). como actitud proposicional cuando la usamos como "rechazo" ${ }^{1}$ o formal en términos de operadores lógico-matemáticos. Otros usos extendidos son el de la negación como cancelación, que entiende que el contenido de un proposición como $\neg \mathrm{A}$, elimina $\mathrm{o}$ cancela el contenido de la proposición expresada por A; la negación como falsedad, que captura la idea de que negar una proposición consiste en afirmar su falsedad; la negación como indicador de inconsistencia, según este abordaje el operador negación nos indica que de la fórmula a que se le aplica se sigue un absurdo (este es el uso que modela la Lógica Intuicionista). Por otra parte, tenemos el enfoque de la negación como indicador de contradictoriedad, el cual establece que una fórmula y su negación son contradictorias, y el operador es entonces un formador de contradicciones. Tenemos también la concepción de la negación como complementación, es decir, negar A es realizar una afirmación que vale justamente cuando A falla ${ }^{2}$. Algunos teóricos 
identifican la negación como un operador de falsedad, que representa por sí mismo este valor (Cfr. Beall, 2017). Finalmente tenemos una manera de entender la negación simplemente como inversión donde negar A es solamente afirmar que las cosas son a la inversa (Cfr. Varzi; Warglien, 2003), es decir que se ofrece el reverso del contenido o valor afirmado.

A diferencia de lo que ocurre con la disyunción o la conjunción, que de manera general modelan aproximadamente los mismos fenómenos lingüísticos, a saber, los de conectar conjuntiva o disyuntivamente dos contenidos, la negación como vimos recoge una serie de mecanismos plurales que no se agotan en uno u otro de los enumerados anteriormente, sino que como en el caso de la Lógica Clásica, un único conectivo condensa una serie de funciones diversas relativas a las prácticas de hablantes y agentes racionales, cuyos efectos impactan sobre la definición de la relación de consecuencia lógica. Usualmente la relación de consecuencia o implicación suele definirse como la ausencia de contraejemplo a una inferencia, esta ausencia de contraejemplo se expresa precisamente como la negación de la existencia de una valuación que viole las condiciones preestablecidas por el sistema (preservación de verdad/ valor designado). Existe una interdependencia entre la definición de la relación de consecuencia lógica, la concepción respecto de los valores de verdad de los que dispone un sistema y la definición del operador de negación en el mismo, que pone un peso extra tanto conceptual como formal en dicho operador a diferencia de los otros dos miembros de la tríada.

A medida que se fueron desarrollando otros sistemas lógicos abriendo el camino de las lógicas no clásicas surgió la cuestión de si es lícito hablar de "la negación" en lugar de "una negación", surgiendo así la posibilidad de tener varios tipos de negación categorizadas según las leyes que obedezca el conectivo dadas las características del sistema en que se halle inserto. Partiendo de la negación clásica como modelo trataremos de contrastar dos operadores de negación alternativos para elucidar que tipo de expectativas debería satisfacer un operador que queramos definir como la negación dentro de nuestros sistemas.

\section{Negación clásica. Exhaustividad y exclusividad.}

La Lógica Clásica (LC) es asociada generalmente con el Principio de Bivalencia por el cual se rige y que dicta que toda fórmula del lenguaje es verdadera o falsa. Es decir, para toda fórmula A, esta es o bien (únicamente) verdadera o bien (únicamente) falsa. Por otro lado, al ser la Lógica Clásica un sistema bivaluado, que cuenta solamente con los valores verdadero y falso respectivamente, estos resultan ser valores contrapuestos y mutuamente excluyentes, de forma tal que cada fórmula debe tener al menos uno y como máximo uno de estos valores. Estas características que hacen a la clasicalidad del sistema y de sus conectivos las encontramos en la literatura rotuladas como:

1)Exhaustividad: toda fórmula del lenguaje adquiere un valor $y$

2)Exclusividad: no es posible que una fórmula tenga a la vez ambos valores, los valores se asignan de manera exclusiva.

Las condiciones de Exhaustividad y Exclusividad se reflejan como restricciones sobre la negación, las cuales se expresan mediante la validez de los principios conocidos como Tercero Excluido (LEM) y EFSQ, también conocido como Explosión. Así la negación cumple con el requisito clásico de exhaustividad validando $\vDash A \vee \neg A$, de manera que para toda fórmula o ella o su versión negada sean verdaderas y con $A \wedge \neg A \vDash C$ restringe la asignación de verdad/falsedad de forma tal que si se solapan el sistema 
se trivializa, o explota. En ambos casos vemos que se han superpuesto las nociones de fórmula asertada/negada con las nociones de verdad y falsedad, esta identificación se verá cuestionada en los sistemas multivaluados donde aparecen valores de verdad intermedios, pero en este caso, como veremos más adelante, algunas de las condiciones anteriores 1 o 2 deberán abandonarse.

Ahora bien, desde el punto de vista algebraico, la Lógica Clásica tiene su correlato en las álgebras de Boole. Un álgebra booleana es una estructura $(A, \wedge, \vee, \neg, 1,0)$ en ella la conjunción y la disyunción se definen como las operaciones binarias de meet/join o ínfimo/supremo para cualesquiera dos elementos, y la negación se define como la operación unaria llamada complemento que cumple que para toda fórmula $a \in \mathrm{A}$, a $\wedge \neg \mathrm{a}=0$ y a $\vee \neg \mathrm{a}=1$. Para Varzi y Warglien esta operación complemento, puede leerse como una de las técnicas que modela la intuición de la negación como forma de complementación, de ofrecer un contenido complementario allí donde el contenido afirmado no vale, no se sostiene ${ }^{3}$. Una particularidad de estas estructuras es que el complemento es único, es decir cada elemento no puede tener más de un complemento, luego el resultado de aplicar el operador negación es un único elemento en cada caso, y dado que sólo comprende dos valores de verdad estos son a su vez el máximo y el mínimo del conjunto ordenado, y en tanto tales no solo conceptualmente sino algebraicamente opuestos.

Este operador de negación clásico satisface también un tercer requisito, la Ley de Doble Negación

$$
\text { 3) } L D N: \neg \neg A \vDash A \text { y } A \vDash \neg \neg A
$$

Vemos que así se termina de completar el círculo conceptual respecto a las condiciones 1 y 2 , en tanto si tenemos dos únicos valores que conmutan de a uno por vez, la iteración de la conmutación nos volverá al valor inicial, o dicho simplemente: si afirmamos que es falso que una fórmula sea falsa estamos afirmando que es verdadera.

La cuarta condición que cumple el operador clásico es la que conocemos como De Morgan:

$$
\text { 4) } L D M: \neg(A \vee B) \Leftrightarrow 4{ }^{4}(\neg A \wedge \neg B) \text { y } \neg(A \wedge B) \Leftrightarrow(\neg A \vee \neg B)
$$

4. Este bicondicional debe entenderse como una equivalencia metateórica, que establece que ambas fórmulas conectadas por él poseen el mismo valor de verdad.

5. En Lenzen (1998) el autor arguye que Contraposición es el requisito que define a la negación per sé. Al no compartir su posición la presentamos en su manera usual como una ley del sistema LC donde el conectivo tiene un rol fundamental.
Esta ley es fundamental porque nos permite establecer la interdefinibilidad de los conectivos conjunción y disyunción por vía de la negación, o si se quiere por vía del establecimiento de sus condiciones de verdad y falsedad mediante el operador en cuestión. Finalmente tenemos además la ley conocida como Contraposición donde a través de la negación obtenemos una equivalencia fundamental para el condicional material:

Contraposición 1: $A \Rightarrow B \Leftrightarrow \neg B \Rightarrow \neg A$.

Dado que en LC el condicional material es interdefinible con la consecuencia lógica obtenemos:

Contraposición 2: $A \vDash B \Leftrightarrow \neg B \vDash \neg A$,

Esto enriquece la lectura de la relación de consecuencia lógica no ya únicamente como preservación de verdad (de izquierda a derecha) sino como preservación de falsedad (de derecha a izquierda). Como se observa la carga que descansa sobre el operador de negación, a pesar de ser el más básico (de menor aridad) del lenguaje, es mayor que la del resto de los conectivos en cuanto a funciones que exceden el mero rol sintáctico de construcción de fórmulas. ${ }^{5}$ 
Si volvemos a las maneras de entender la negación que enumeramos al principio vemos como algunas de ellas son recogidas en las condiciones de Exhaustividad y Exclusividad, así como otras encuentran un correlato en la definición algebraica de la operación complemento de la estructura booleana. Podemos decir que este conectivo logra modelar exitosamente muchos de los mecanismos de oposición que caracterizan de manera amplia nuestra noción intuitiva de negación, así como aquellas ideas de negación como rechazo, incompatibilidad (de contenido o de valores de verdad), contradictoriedad, marcador de falsedad y conmutador de valores. Esta negación clásica cuyas normas principales listamos en 1-4 es conocida también como negación "estricta" o "fuerte". Más adelante volveré sobre esta noción mostrando que es posible deshacer esta identificación, y definir un operador de negación "estricto" sin que necesariamente sea una negación clásica.

\section{La negación en sistemas no clásicos. K3, LP y FDE}

Durante las últimas décadas han crecido considerablemente los esfuerzos teóricos dirigidos a la creación de nuevos sistemas lógicos que se alejen de los cánones de la Lógica Clásica y que puedan resolver algunos problemas que LC no resuelve, ya sean de tipo práctico, en el trabajo con ciertos sistemas de información, o de tipo teórico, el trabajo con vista a solucionar paradojas semánticas, conjuntistas o de otros tipos. Este es el caso de los sistemas lógicos multivaluados, es decir que abandonan el paradigma de la bivalencia para incluir terceros y hasta cuartos valores de verdad. Las novedades aportadas por estos sistemas tienen más peso en el trabajo de la negación, como observa Lenze(1998), ya que muchos de ellos tratan de recapturar casi totalmente el funcionamiento clásico de la conjunción y la disyunción, hasta donde es posible.

El caso de las lógicas $K_{3}$ y LP, es uno de los ejemplos más relevantes en esta área. Ambas utilizan el esquema de valuación conocido como Kleene fuerte (Cfr. Priest, 2008), el cual amplía el conjunto de valores de verdad de forma que $\mathrm{V}=\left\{1,0 \mathrm{y}^{1 / 2}\right\}^{6}$. La interpretación filosófica que podamos dar a este tercer valor $1 / 2$ dependerá del sistema lógico en que nos encontremos. En el caso de LP, sistema desarrollado por Graham Priest para modelar el trabajo con paradojas semánticas, de ahí su nombre Logic of Paradox, nos encontramos con un sistema paraconsistente donde podemos leer este tercer valor como Both(B) o como Glut, expresando una asignación de valores de verdad no exclusiva. Así las fórmulas con valor b podrán ser interpretadas como "verdaderas y falsas" simultáneamente o cúmulos (Gluts) de valores de verdad. La característica fundamental de este tipo de sistema es el rechazo del principio de explosión o EFSQ, este rasgo es el que lo caracteriza como paraconsistente ${ }^{7}$, de forma que obtenemos:

$$
A \wedge \neg A \not{ }_{L P} B
$$

En este sentido la negación paraconsistente no cumple con la condición 2, ya que permite que pueda asignarse el mismo valor de verdad a una fórmula y a su negación, esto hace que el sistema pueda tolerar contradicciones sin que por ello implique trivialidad. Luego, en el caso de negar una fórmula que tome el valor $1 / 2$ tendremos que:

$$
v(A)_{L P}=v(\neg A)_{L P}=1 / 2 \text {. }
$$

Digresión: Un detalle ineludible al respecto del operador negación de LP es que su presentación por parte de Priest viene aparejado a una interpretación filosófica particular denominada dialetheismo. El dialetheismo es la posición que sostiene a nivel semántico que algunas oraciones son verdaderas y falsas y a nivel más general, ontológicamente hablando, que algunas contradicciones son verdaderas. Como se observa
6. Utilizamos 1 y T para representar la verdad, así como 0 y $\mathrm{F}$ para la falsedad.

7. Existe un debate al respecto de la caracterización definitiva de un sistema o conectivo como paraconsistente. Cfr Barrio \& Da Ré (2018). 
8. Para otra instancia de refutación de la idea de "interpretación canónica" aplicado a sistemas paraconsistentes ver Barrio (2018). esta posición descansa fundamentalmente en el funcionamiento del operador de negación de dicho sistema, que en primer lugar bloquea explosión, permitiendo que dichas contradicciones verdaderas interactúen dentro del sistema sin trivializarlo, y por otro lado que le adjudica una lectura según la cual este operador forma contradicciones que de alguna manera no presuponen para su contradictoriedad la exclusividad de los valores de verdad/falsedad. Una vez más se pone de relieve como la definición de un operador negación y su lectura filosófica asociada tiene un impacto definitivo sobre las lecturas posibles de los valores de verdad y hasta sobre una ontología posible a esbozar a partir de estos, i.e si existen o no objetos contradictorios que satisfagan las afirmaciones basadas en dialetheias.

De cualquier manera seguimos a Barrio y $\mathrm{Da} \operatorname{Re}(2018)$ cuando consideramos que el dialetheismo es una interpretación filosófica posible, o una motivación entre otras, para adoptar una lógica y por extensión un operador de negación paraconsistente, y que ambos fenómenos no están conectados necesariamente ni sujetos a una identificación formal o conceptual. Por el contrario, observamos que de manera general la idea de "interpretación canónica" de un sistema lógico, a saber, que existe una única interpretación filosófica o lectura adecuada para cada sistema lógico cuenta con innumerables contraejemplos, y es más una expresión de nuestros deseos como teóricos que una característica formal o conceptual que pueda de hecho derivarse de los rasgos fundamentales de cualquier sistema. ${ }^{8}$ Luego, en este trabajo me ocupo sólo de la noción formal de paraconsistencia centrada en el papel fundamental que tiene el operador negación para definir su rasgo principal, i.e. invalidar la inferencia Explosión, dado que mi objetivo es argumentar con la mayor generalidad posible sobre el uso del conectivo y no centrarme únicamente en una interpretación particular de dichos sistemas. Fin de digresión

Volviendo sobre los sistemas trivaluados construidos sobre Kleene fuerte, encontramos como contrapartida de LP en el sistema K3 tenemos que el operador negación no cumple con la condición 1, de forma que el Principio de Tercero Excluido no resulta una fórmula válida, es decir:

$$
\not_{\mathrm{k} 3} \mathrm{~A} \vee \neg \mathrm{A}
$$

La falla de este principio es lo que otorga al sistema el rótulo de paracompleto, y el tercer valor $1 \frac{1}{2}$ es leído como Neither $(\mathrm{N})$ o como Gap, es decir como un vacío de verdad, aquellas fórmulas que tomen su valor podrán ser consideradas como "ni verdaderas ni falsas". Ahora bien, el operador de negación en $\mathrm{K}_{3}$, tal como en LP mantiene su funcionamiento standard para valores clásicos, i.e. si $\mathrm{v}(\mathrm{A})=1$ entonces $\mathrm{v}(\neg \mathrm{A})=0$ y viceversa, pero en caso del tercer valor tenemos que:

$$
v(A)_{K 3}=v(\neg A)_{K 3}=1 / 2 .
$$

Es decir, para el caso del tercer valor la negación retorna al mismo valor que toma como argumento perdiendo así la función de conmutador de valores que habíamos señalado anteriormente.

De manera general suele rotularse a estas negaciones que siguen el esquema de valuación de Kleene como "negaciones débiles" ya que fallan en cumplir el mecanismo de oposición de valores para todos los valores disponibles, y el tipo de oposición que logra modelar es difícilmente la oposición de contradictoriedad, al menos entendida en el sentido estricto de imposibilitar que una fórmula y su negación sean verdaderas simultáneamente (Cfr. Arenhart $\&$ Melo, 2017). De cualquier manera hay algún desacuerdo sobre si el rótulo "débil" debe reservarse sólo para las negaciones que no cumplen EFSQ o si debe también aplicarse a aquellas que evaden LEM (Cfr. Lenzen, 
1998), pero de manera general podemos decir que se evidencia cierta anomalía que podríamos nombrar laxamente como "debilidad" para los casos en que el operador no cumple los requisitos 1 y 2 y además hay valores para los cuales una vez aplicado retorna el mismo valor que toma como input.

Veamos ahora el caso particular de otro sistema no clásico, FDE. El sistema que toma las siglas de First Degree Entailment fue presentado por Anderson y Belnap durante la década del '70 como un sistema aplicado para el trabajo de programación en áreas de inteligencia artificial. El resultado es un sistema cuatrivaluado cuyo conjunto de valores de verdad se amplía hasta $V=\{0, b, n, 1\}$. Por una parte, este sistema nos permite mayor libertad que los dos anteriores ya que al incluir tanto el valor Glut como el valor Gap permite brindar tratamientos diferentes a diferentes tipos de fenómenos teóricos o prácticos. Por ejemplo, podríamos acomodar tanto soluciones paraconsistentes como paracompletas a distintas paradojas semánticas. Una vez más tenemos en FDE el operador de negación funcionando como conmutador para los valores clásicos, pero para los valores no clásicos, tanto b como n, tenemos que opera à la Kleene, es decir:

$$
\text { Si v }(A)_{F D E}=b \text { entonces } v(\neg A)_{F D E}=b \text { y si } v(A)_{F D E}=n \text { entonces } v(\neg A)_{F D E}=n \text {. }
$$

En FDE, además, tanto los principios EFSQ como LEM resultan inválidos, es decir su negación no cumple los requisitos 1 y 2 sin embargo, debemos notar que 3 sigue siendo válida tal como para K3 y LP. Luego la situación es bastante similar a la anterior mencionada en cuanto a la debilidad del operador, y las mismas críticas le caben en cuanto a si de hecho cumplen con la intuición preteórica de la negación como algún tipo de mecanismo de oposición que debe modelarse a través de la permutación de valores.

Por último, mostramos la representación gráfica de los dos órdenes más explorados en la literatura a partir de FDE. El orden de la verdad o $\mathrm{T}$ ( $\left.\mathrm{f}_{1}\right)$ y el orden de la información o I (f2).

f1

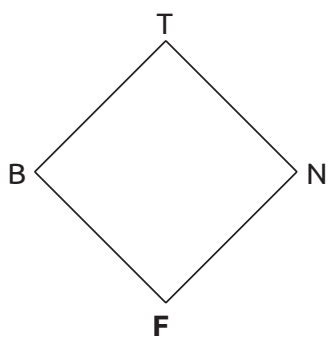

f2

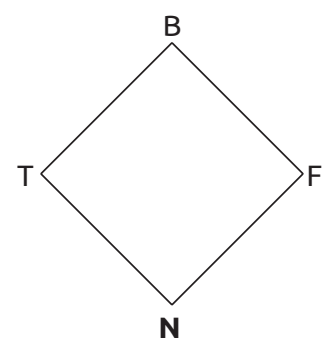

\section{La propuesta de Omori y De: BD+. Alcances y límites}

En su texto "Classical negation and expansions of Belnap-Dunn logic" De Michael \& Omori (2015) proponen un análisis de la negación clásica desde una perspectiva no clásica. Su propósito es rastrear la posibilidad de la definición de un operador de negación clásico en sistemas no clásicos con matrices multivaluadas. Para esto brinda una caracterización de la negación clásica usando herramientas semánticas ya que según él no es posible solo con herramientas sintácticas capturar aquello que esencialmente podría elucidar la clasicidad de la negación. Dado que el autor persigue una definición de clasicidad para todo el espectro no-clásico, incluyendo sistemas trivaluados y cuatrivaluados, el propósito de máxima generalización le lleva a añadir o modificar requisitos al operador según el tipo de matriz, requisitos semánticos que 
dicho operador debe cumplir y que el autor rotula en tres propiedades: "Toggle", "Contra" "Liberal" como su generalización a contextos trivaluados) e "Involution". Veamos de qué se trata:

1) Toggle: Una operación sobre valores de verdad se considera una negación clásica sólo si conmuta (toggles) entre los valores clásicos T/F, i.e. si lleva T a F y a la inversa (De Michael \& Omori, 2015).

2) Contra: $A V B$ son clásicamente contradictorios sii $A \bigvee B$ es siempre verdadero y no falso ${ }^{9}$ y $A \wedge B$ es siempre falso y no verdadero.

3) Involution: Una operación $\otimes$ sobre valores de verdad i es una negación clásica solo si $\otimes \otimes \mathrm{i}=\mathrm{i}$. Es decir, si vale la equivalencia semántica de una oración con su doble negación.

Si bien su propósito es generalizar la definición del operador clásico para todos los contextos no clásicos a continuación nos enfocamos en su propuesta para un sistema cuatrivaluado por ser el tipo de sistema que nos ocupa.

Omori y De extienden el sistema FDE con lo que denominan una negación clásica obteniendo BD+. Esta negación una vez que le aplicamos los requisitos semánticos 1, 2 y 3, resulta ser una negación booleana por definirse como la operación compleclásicos verdad/falsedad como un par, es decir de manera simétrica en sus condiciones de verdad y falsedad:

$\neg \mathrm{A}$ is verdadero sii $\mathrm{A}$ es no verdadero.

$\neg$ A es falso sii A es no-falso.

El resultado es un operador que se comporta de la manera siguiente:

\begin{tabular}{c|c}
$\neg$ & \\
\hline 1 & 0 \\
\hline $\mathrm{o}$ & 1 \\
\hline $\mathrm{b}$ & $\mathrm{n}$ \\
\hline $\mathrm{n}$ & $\mathrm{b}$
\end{tabular}

f3

Sin embargo, como reconoce el mismo autor, esta noción desplaza el peso de la clasicidad de la negación hacia las definiciones de $\wedge$ y V (Cfr. De Michael \& Omori, 2015: 828). Es decir, la clasicidad de la negación descansa sobre la definición standard de los conectivos $\wedge$ y $\vee$ como meet y join que le sirven para definir su noción de Contradictoriedad. Es decir, la negación resulta clásica porque se apoya en dos conectivos definidos sobre operaciones también clásicas. No debe sorprendernos que el resultado de esta interacción coincide con LC.

Por otra parte, si bien este operador que nos proponen Omori y De cumple con los requerimientos que plantea para una negación clásica no brinda una fundamentación para la permutación entre los valores no clásicos b y n, la cual aparece únicamente como un resultado de la operación de complementación sobre el retículo, es decir un efecto secundario de identificar la noción de clasicidad de la negación con la negación booleana. Sin embargo, como mencionamos anteriormente otros órdenes son posibles 
sobre el conjunto FOUR donde el orden informacional de los valores nos presenta una lectura filosófica mucho más rica al respecto de la oposición de dichos valores b y n en términos de contenido informativo, conteniendo b el punto de máxima información y n el mínimo, lo cual hace que resulte más intuitivo el funcionamiento de conmutador que el operador tiene ante estos valores. ¿Es esto suficiente para elegir entonces el orden de la información sobre el orden de la verdad? Mi opinión es que no, dado que uno $\mathrm{u}$ otro orden son similares en cuanto a la definición de los operadores $\Lambda \mathrm{y}$ $V$, en tanto en uno los elementos clásicos resultan incomparables (I) y en el otro (T) ocurre con los no clásicos. A continuación, ampliamos sobre esta particularidad y su posible repercusión en la interpretación de ciertas valuaciones.

\section{Una negación estricta para un sistema cuatrivaluado}

En uno de sus textos recientes Beall (2016) postula a FDE como una solución "natural" al dilema de si ante la necesidad de abandonar la lógica clásica deberíamos optar por un sistema o bien paraconsistente o bien paracompleto. FDE nos ofrece según él la posibilidad de encontrar un equilibrio entre ambas opciones y tener un sistema que pueda tolerar ambos enfoques teóricos de Gluts y Gaps de verdad. Este argumento parece plausible en tanto parece alcanzarse lo que Beall llama un "equilibro teórico" cuando modelamos dichos fenómenos con el conjunto FOUR, pero a diferencia del autor no creo que el sistema adecuado sea FDE en ninguno de sus dos órdenes T e I. Sin embargo, es posible diseñar otro tipo de sistema cuatrivaluado a partir de este mismo conjunto de valores.

Rescatemos en primer lugar qué características debe tener una negación en este tipo de sistema. Por un lado, es fundamental que conserve su función de conmutador de valores para todo el espectro de valores disponibles. Esta característica en general suele dar al operador de negación el rótulo de "negación fuerte" o "negación estricta" en oposición a las "negaciones débiles" cuya función de switch solo opera para valores clásicos. Sin embargo, no es necesario que dicha negación estricta sea a la vez clásica. Podemos definir una negación fuerte que cumpla las siguientes propiedades:

1) Flip-flop: Permutar entre los valores opuestos 1 y 0 / B y $\mathrm{N}$.

2) LDN: Cumplir la equivalencia semántica entre una proposición y su doble negación. i.e. que se cumpla para toda proposición $A$ que $\neg A \equiv \neg \neg A$.

3) Leyes de De Morgan: Hacer válidas las siguientes equivalencias:

$$
\neg(A \wedge B) \equiv(\neg A \vee \neg B) \quad \neg(A \vee B) \equiv(\neg A \wedge \neg B)
$$

En el caso de 1 vemos que es un poco más exhaustiva que Toggle. Es decir, queremos no solo que el operador de negación conmute clásicamente, queremos que lo haga para todo el espectro de valores. Este requisito obedece al hilo fundamental de nuestra argumentación respecto del operador negación como aquel que captura los mecanismos de oposición más generales de nuestra práctica lingüística y racional. A su vez incluimos la LDN no solo por ser altamente intuitiva y estar en correspondencia con las prácticas habituales de los hablantes sino porque junto a Flip Flop constituyen las dos caras de una misma función que hacen a la fortaleza del operador: la posibilidad de seleccionar dos elementos opuestos semánticamente como pares y conmutar entre ellos aisladamente, en un contexto donde existen otros dos valores que podrían considerarse como alternativas. Ambas funciones establecen un contrapeso entre sí, la una recoge un mecanismo de oposición semántica y la otra establece la equivalencia semántica entre una oración y la iteración de esta oposición. Por otra 
parte, las Leyes de De Morgan no solo son parte de la ortodoxia en cuanto a los requisitos de las negaciones estrictas, canónicamente la negación clásica, sino que además permiten mostrar la prioridad del operador con respecto a la disyunción y la conjunción sobre las que puede distribuirse (no así a la inversa) garantizando de esta forma la interdefinibilidad de los mismos.

Sin embargo, esta función de conmutador es deseable que opere con cierta estabilidad, es decir, en palabras de Omori, que no devuelva un output no clásico cuando recibió un input clásico, y agregamos, que tampoco ocurra lo inverso. Para explicar este punto podemos volver sobre la extensión $\mathrm{BD}+$ que agrega a FDE una negación clásica. Como vimos dicha negación cuya característica fundamental es la Contradictoriedad descansa sobre una noción clásica de $\Lambda$ y $\vee$, digamos que su clasicidad se desplaza hacia la de estos operadores.

Una de las consecuencias de este desplazamiento es que debemos atenernos a los resultados de aplicar dichas definiciones de $\wedge$ y $\vee$ sobre el lattice de diamante de FOUR con el orden $\mathrm{T}$, donde la interacción de la conjunción y la disyunción entre valores no clásicos nos arroja un resultado clásico $(\mathrm{n} / \mathrm{b}=0 / \mathrm{nVb}=1)$. Una pregunta relevante es ¿por qué deberíamos aceptar este tipo de interacciones? Si como el autor explica al justificar su elección de Toggle como un requisito fundamental, no es deseable un operador de negación que devuelva outputs no clásicos para inputs clásicos, ¿por qué deberíamos aceptar la inversa? y ¿por qué no podemos extender este requisito de la negación para el resto de los conectivos? Si nos resulta contraintuitivo pensar que la negación de una oración como "La nieve es blanca" pueda tener un valor no clásico, es discutible que deba aceptarse que la negación de una oración como la Paradoja del Mentiroso deba ser simplemente falsa, o simplemente verdadera. De la misma forma parece discutible que una disyunción entre oraciones con valores como b y n pueda evaluarse como simplemente falsa o simplemente verdadera, pensemos el siguiente ejemplo:

L: "Esta oración es falsa."

F: "Mañana habrá una batalla naval en el puerto de Buenos Aires."

P: “Esta oración es falsa o mañana habrá una batalla naval en el puerto de Buenos Aires"

Supongamos que asignamos a $\mathrm{L}$ el valor $\mathrm{b}, \mathrm{y}$ a $\mathrm{F}$ el valor $\mathrm{n}$ como ocurre frecuentemente. No parece haber ningún motivo más allá de la definición algebraica del operador $V$ sobre el lattice de orden $T$ para que esta disyunción sea simplemente verdadera, como ocurre en FDE y en BD+. Otro tanto ocurriría si aplicamos a L y F la operación $\Lambda$ cuyo resultado sería $o$. Nos parece adecuado entonces añadir un requisito general en el contexto de un sistema cuatrivaluado.

Llamémosle a esta propiedad de mantener la estabilidad entre la clasicalidad (o no clasicalidad) de los valores de verdad entre los inputs y los outputs de las operaciones lógicas "regularidad". Esta característica la encontramos tanto en los operadores de negación no clásicos de LP y K 3 , como en la negación original de FDE y en la negación booleana con que Omori y De extienden la lógica de Belnap y Dunn

Regularidad: Un operador lógico $\otimes$ es regular si mantiene la clasicalidad o no clasicalidad entre sus inputs y sus outputs. i.e. $\otimes T / F=T / F \otimes B / N=B / N$ o bien $T \otimes F=T / F B \otimes N=B / N$.

A simple vista se observa que la negación estricta que formulamos se comporta regularmente. Para el caso de la disyunción y la conjunción podemos ofrecer las siguientes 
valuaciones donde en los casos mixtos lo que ha jugado el rol decisivo ha sido nuestra comprensión de los valores como completamente interrelacionados, es decir sin valores incomparables, pero respetando la definición de conjunción como meet (ínfimo) y disyunción como join (supremo) o de un modo más intuitivo aquellas definiciones donde el resultado de la conjunción de dos valores tiende al mínimo y la disyunción al máximo de los dos.

De esta manera obtenemos las siguientes tablas de verdad para $\Lambda$ y $\vee$.

\begin{tabular}{c|c|c|c|c}
$\wedge$ & 1 & $\mathrm{~b}$ & $\mathrm{n}$ & $\mathrm{o}$ \\
\hline 1 & 1 & $\mathrm{~b}$ & $\mathrm{n}$ & $\mathrm{o}$ \\
\hline $\mathrm{b}$ & $\mathrm{b}$ & $\mathrm{b}$ & $\mathrm{n}$ & $\mathrm{O}$ \\
\hline $\mathrm{n}$ & $\mathrm{n}$ & $\mathrm{n}$ & $\mathrm{n}$ & $\mathrm{o}$ \\
\hline $\mathrm{o}$ & $\mathrm{o}$ & $\mathrm{o}$ & $\mathrm{o}$ & $\mathrm{o}$
\end{tabular}

\begin{tabular}{c|c|c|c|c}
$\mathrm{V}$ & 1 & $\mathrm{~b}$ & $\mathrm{n}$ & $\mathrm{O}$ \\
\hline 1 & 1 & 1 & 1 & 1 \\
\hline $\mathrm{b}$ & 1 & $\mathrm{~b}$ & $\mathrm{~b}$ & $\mathrm{~b}$ \\
\hline $\mathrm{n}$ & 1 & $\mathrm{~b}$ & $\mathrm{n}$ & $\mathrm{n}$ \\
\hline $\mathrm{o}$ & 1 & $\mathrm{~b}$ & $\mathrm{n}$ & $\mathrm{o}$
\end{tabular}

Dichas valuaciones pueden graficarse con un retículo en forma de cadena $u$ orden total, cuyos elementos se encuentran ordenados tal que $\mathrm{V}=\{0<\mathrm{n}<\mathrm{b}<1\}$ como se muestra a continuación.

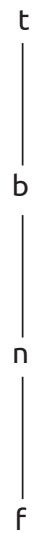

De esta forma se mantiene la definición standard de $\Lambda$ y $V$ como meet y join pero con resultados regulares, i.e. $b \bigvee n=b$ y $b / n=n$. También podemos comprobar que para el caso de los elementos o y 1 , se cumple que uno es el complemento del otro, retomando el aire de familia con LC para el subconjunto de valores clásicos. Finalmente, al ser la estructura un retículo distributivo se cumplen las leyes de De Morgan. Otro tanto puede corroborarse con LDN ya que para toda fórmula A se cumple que $\mathrm{v}(\mathrm{A}) \equiv \mathrm{v}(\neg \neg \mathrm{A})$.

Ahora bien, para verificar que estas valuaciones no nos devuelven a la Lógica Clásica como en el caso de $\mathrm{BD}+$ podemos definir una relación de consecuencia lógica que, en sintonía con el principio de tolerancia acomode tanto a teóricos glutty como gappy, permita valuar a los fenómenos paradójicos tanto b como n y en tanto tal permita tener ambos valores como designados. Podemos definir entonces a esta relación $\vDash$ como preservación de valor designado y al conjunto de valores designados como $\mathrm{D}$ $=\{\mathrm{n}, \mathrm{b}, \mathrm{1}\}$. De esta forma además obtenemos un contraejemplo a Explosión, lo que brindará un entorno propicio para abordajes paraconsistentes.

A saber: para $\mathrm{A} \wedge \neg \mathrm{A} \vDash \mathrm{B}$ existe una valuación $\mathrm{v}$ tal que: $\mathrm{v}(\mathrm{A})=\mathrm{b}$ y $\mathrm{v}(\mathrm{B})=0$, luego $\mathrm{v}(\neg \mathrm{A})=\mathrm{n}, \mathrm{y}$ así $\mathrm{v}(\mathrm{A} \wedge \neg \mathrm{A})=\mathrm{n}$ y corroboramos la pérdida de valor designado de $\mathrm{n}$ a o. Luego $\mathrm{A} \wedge \neg \mathrm{A} \not \mathrm{B}$.

Debe notarse que en el caso de LEM, este principio resulta válido, es decir obtenemos $\vDash A \bigvee \neg A$ para toda fórmula del lenguaje, esta pequeña desventaja para el teórico gappy se equilibra con la posibilidad de valuar oraciones problemáticas con valor $\mathrm{n}$ 
11. Decimos que abandona Exhaustividad en el sentido de que hay más valores posibles que solo verdadero y falso, de hecho, el valor $n$ puede interpretarse como ausencia de valor de verdad. Sin embargo, es cierto que si identificamos exhaustividad con LEM este principio resulta válido y en algún sentido el sistema es exhaustivo, es decir, asigna valor de verdad a toda proposición, solo que las opciones se extienden al espectro de lo no clásico. y obtenerlas como válidas dentro del lenguaje. Recordemos que a pesar de que no es válido Explosión el $\mathrm{PNC}, \vDash \neg(\mathrm{A} \wedge \neg \mathrm{A})$, sí aparece como una fórmula válida. Esta particularidad no nos toma por sorpresa ya que ocurre también en lógicas paraconsistentes construidas sobre matrices de Kleene fuerte como LP. De alguna manera la validez de ambos principios (LEM y PNC) en sintonía con la posibilidad de tener ambos valores como designados restaura el equilibrio teórico que siguiendo a Beall queríamos rescatar al tomar FOUR como base de nuestro sistema.

De esta manera este nuevo operador de negación, si bien es no clásico pues abandona las condiciones de clasicalidad Exclusión y Exhaustividad ${ }^{11}$ cumple las expectativas de modelar lo que de manera global podemos rotular como negación fuerte o estricta, un mecanismo plural de oposición o de inversión de valores y contenidos. Este operador cumple las dos condiciones que pedimos, Doble Negación y Flip Flop, que de manera general se avienen a nuestras intuiciones como hablantes. De la misma manera también cumple la equivalencia establecida por las Leyes De Morgan que establecen la dualidad de los operadores de conjunción y disyunción mediante el funcionamiento de la negación. Hemos definido así un operador que se ajusta a los requisitos básicos que podríamos esperar de una negación fuerte en un contexto cuatrivaluado manteniendo su no clasicalidad.

\section{Conclusión}

En este trabajo realicé un análisis del significado de los operadores de negación contrastando la negación clásica con las negaciones no clásicas paraconsistentes y paracompletas y su funcionamiento en los sistemas K3, LP y FDE. Esbocé una crítica de las negaciones no clásicas que funcionan à la Kleene y propuse como requisito deseable a cumplir por un operador de negación el de ser capaz de conmutar entre los valores de verdad que el sistema tenga disponibles a partir de la idea de oposición como espíritu fundamental del fenómeno que modela. Analicé la propuesta de Omori y De que busca caracterizar una negación clásica en un contexto no clásico y mostré por qué no es del todo satisfactoria. Finalmente propuse tomar como base el conjunto FOUR estableciendo un orden total e incorporando una negación estricta que sea a la vez no-clásica y cumpla con las características mínimas de una negación fuerte: Conmutar entre los valores de verdad, cumplir con la Ley de Doble Negación y validar las Leyes de De Morgan. Finalmente mostré cómo es posible definir una relación de consecuencia que, mediante la inclusión de tres valores designados, permite invalidar Explosión y ratificar la no clasicalidad del sistema. 


\section{Bibliografía}

" Arenhart, J.R.B. \& Melo, E.S. (2017). Classical negation strikes back: why Priest's attack on classical negation can't succeed. Logic Universalis, 11: 465,465-487. doi: 10.1007/s11787-017-0178-z

» Barrio E. Models and Proof: LFIs without a canonical interpretation. Principia, 22 (1), 87-112. doi: 10.5007/1808-1711.2018v22n1p87

" Barrio E. \& Da Ré, B. (2018). Paraconsistency \& Philosophical Interpretations. Australasian Journal of Logic, 15 (2). doi: 10.26686/ajl.v15i2.4860

» Beall, Jc. (2017). There is no Logical Negation: True, False, Both, and Neither. The Australasian Journal of Logic, [S.l.], 14 (1) doi: 10.26686/ajl.v14i1.4025

» De Michael \& Omori H. (2015) Classical negation and expansions of BD. Studia Logica, 103(4), 825-851. DOI: https://doi.org/10.1007/s11225-014-9595-7

» Horn, Laurence R. (1989) A natural history of negation. Chicago: University of Chicago Press.

» Horn, Laurence R. \& Wansing, H. (2017). Negation. En Zalta, E. (Ed.). The Stanford Encyclopedia of Philosophy.

»Lenzen W. (1998). Necessary Conditions for Negation-Operators (with Particular Applications to Paraconsistent Negation). En Besnard P., Hunter A. (Eds). Reasoning with Actual and Potential Contradictions. Handbook of Defeasible Reasoning and Uncertainty Management Systems, vol 2. (pp. 211-239) Dordrecht: Springer. doi: 10.1007/978-94-017-1739-7_7

» Priest, G. (2008). An Introduction to Non-Classical Logic. From If to Is. Cambridge: Cambridge University Press. doi: 10.1017/CBO9780511801174

» Rubin, M., \& Roffé, A. (2018). Against a Metaphysical Understanding of Rejection. Principia: an international journal of epistemology, 22(1), 189-202. doi:10.5007/1808-1711.2018v22n1p189

» Varzi, A. C.; Warglien, M. (2003). The geometry of negation. Journal of Applied Non-Classical Logics 13(1), 9-19. doi: 10.3166/jancl.13.9-19 
